\title{
Interleukin-Hemmer Made in Austria
}

Interleukin-Hemmer haben die Therapie von Autoimmunerkrankungen wesentlich erweitert. Ein Symposium widmete sich der Wirksamkeit von Sekucinumab, einem in Österreich hergestellten Wirkstoff.

Secukinumab ist ein seit 2015 zugelassener Hemmer von Interleukin (IL) 17A. Interleukine sind Botenstoffe, die Informationen zwischen den Zellen des Immunsystems vermitteln und an diversen Abwehrund Entzündungsreaktionen beteiligt sind. Bislang sind über 30 von ihnen bekannt. Ihre immunfördernde Wirkung wird für spezifische Tumortherapien genutzt.

\section{Interleukin-Blockade als Therapie} Die Hemmung von Interleukinen durch Biologika in der Therapie von Autoimmunerkrankungen wie der Psoriasis hat revolutionäre Fortschritte gebracht. Künstlich hergestellte Antikörper, die gezielt Interleukine blockieren, unterbinden effizient fehlgeleitete Immunreaktionen. Das eröffnete Prof. Dr. Dr. Diamant Thaci vom Universitätsklinikum Schleswig-Holstein in Lübeck im ersten Vortrag des Symposiums, in welchem er die Perspektive des Dermatologen mit konkreten Zahlen unterlegte.

Eine Langzeittherapie mit dem Interleukin-17A-Hemmer Secukinumab erzielt zwischen 70 und $80 \%$ Besserung bis hin zur Heilung nicht nur bei den Hautsymptomen der Psoriasis, sondern auch bei assoziierten Krankheitserscheinungen wie Enthesitis (Entzündung der Sehnen- und Bänderansätze am Knochen), der Nagelpsoriasis und der Daktylitis (Fingerentzündung).

Auf die Psoriasis-Arthritis und einen axialen Befall der Wirbelsäule hat Secukinumab ebenso positive Wirkung wie als Folgetherapie bei Wirkverlust zuvor eingesetzter anderer InterleukinHemmer.

\section{》) Eine Langzeittherapie mit Secukinumab erzielt zwischen 70 und $80 \%$ Besserung}

Um arthritische Symptome gar nicht erst aufkommen zu lassen, empfiehlt Prof. Thaci den frühzeitigen Einsatz von Secukinumab als Erstlinientherapie anzudenken. Eine entsprechende Zulassung der Europäischen Arzneimittelbehörde (EMA) für das Medikament liegt bereits vor.

\section{Langzeiterfolge}

Im anschließenden Vortrag über Langzeitdaten der Biologika-Therapie bei ankylosierender Spondylitis sprach sich auch Prof. Dr. Jürgen Braun vom Rheumazentrum Ruhrgebiet der Ruhr Universität Bochum für eine frühe Behandlung von Entzündungssymptomen aus, damit strukturelle und also röntgenologisch sichtbare Krankheitserscheinungen gar nicht erst entstehen.

Den von ihm präsentierten MEASURE-Studien zufolge ist Secukinumab unabhängig vom CRP-Wert der Patienten wirksam und zeigt auch in der Langzeittherapie über vier Jahre konstant positive Effekte. Langfristig ist unter dem IL-17A-Hemmer eine nur geringe Röntgenprogression der ankylosierenden Spondylitis festzustellen.

Auch in puncto Sicherheit liefert Secukinumab gute Ergebnisse. Selbst bei langfristiger Therapie ist keine Zunahme von Nebenwirkungen wie entzündlichen Darmerkrankungen,
Neutropenie oder Tumorentwicklung festzustellen.

Über einen langen Zeitraum betrachtet, so Braun, würde sich aber auch zeigen, dass nicht alle Biologika überall gleich wirksam sind. In der vielversprechenden Anti-Interleukin-Therapie gegen Autoimmunerkrankungen sei das letzte Wort noch nicht gesprochen.

》) Eine frühe Behandlung von Entzündungssymptomen beugt strukturellen Krankheitserscheinungen vor

Die Moderation der Veranstaltung führte Prim. Prof. Dr. Josef Smolen, Leiter der Rheuma- und der Früharthritis-Ambulanz am AKH Wien. Sein abschließender Vortrag verglich Daten von Biologika aus den USA und der EU, die hier wie dort eine große Variabilität zwischen den Medikamenten-Chargen zeigen. Mit Smolens Betrachtungen über Ähnlichkeiten bzw. Unterschiede von Originator-Medikamenten und nachahmenden Biosimilars und mit einer kurzen Diskussion, in welcher der stattfindende Preisverfall von Biologika zur Sprache kam, schloss das Symposium und machte im Vortragssaal des Tech Gate Vienna Platz für die nächste Veranstaltung im Rahmen der Jahrestagung der ÖGR.

rheuma plus $2019 \cdot 18: 64$ https://doi.org/10.1007/s12688019-0242-2

C Springer-Verlag GmbH Austria, ein Teil von Springer Nature 2019 